\title{
Chlorophyll- and anthocyanin-rich cell organelles affect light scattering in apple skin
}

\author{
Stefan A. Lohner ${ }^{1}\left(\mathbb{0} \cdot\right.$ Konni Biegert $^{2} \cdot$ Ansgar Hohmann $^{1} \cdot$ Roy McCormick $^{2} \cdot$ Alwin Kienle $^{1}$
}

Received: 28 September 2021 / Accepted: 21 December 2021 / Published online: 9 January 2022

(c) The Author(s), under exclusive licence to European Photochemistry Association, European Society for Photobiology 2022

\begin{abstract}
Apple skin contains several groups of strongly absorbing cell organelles with pigments that change dynamically in type and concentration during fruit maturation. Chlorophylls and carotenoids, both primarily involved in photosynthesis, are found in the grana of chloroplasts, while anthocyanin vacuolar inclusions (AVIs) accumulate for light protection in red-skinned cultivars. A Mie model describing light scattering by absorbing spherical particles in a non-absorbing medium allowed to theoretically investigate the explicit influence of grana and AVIs on the effective scattering coefficient $\mu_{s}^{\prime}$ and the absorption coefficient $\mu_{a}$. The reconstruction of the complex refractive indices of the organelles predicted anomalous dispersion, i.e., a local increase in the real part of the refractive index in the spectral regions with high chlorophyll and anthocyanin absorption, in agreement with the Kramers-Kronig relations. As a result, peaks in $\mu_{s}^{\prime}$ were predicted to be shifted to longer wavelengths compared to the corresponding $\mu_{a}$ bands. This selective scattering effect was confirmed experimentally with integrating sphere measurements for red- or green-skinned apple samples of the cultivars 'Elstar', 'Gala' or 'Jonagold'. Comparison between simulations and measurements indicated that the Soret bands of chlorophyll $a$ and chlorophyll $b$ are at $435 \mathrm{~nm}$ and $469 \mathrm{~nm}$, respectively, and overlap with the absorption of carotenoids, whose red-most edge is at $488 \mathrm{~nm}$. For anthocyanin absorption, a pronounced blue shift from 550 to $520 \mathrm{~nm}$ was observed, indicating structural or chemical changes of AVIs.
\end{abstract}

\section{Graphical abstract}
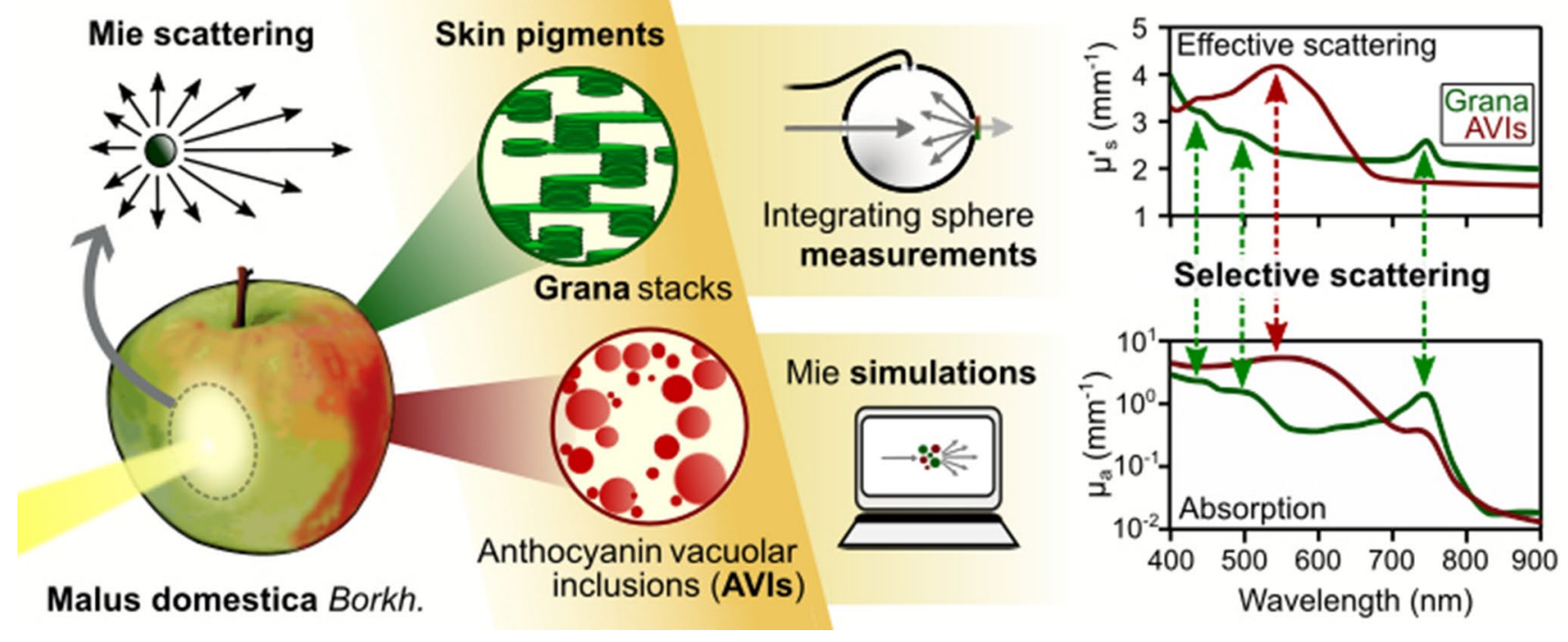

Keywords Selective scattering $\cdot$ Mie's theory $\cdot$ Grana $\cdot$ Anthocyanin $\cdot$ Carotenoids

Stefan A. Lohner

stefan.lohner@ilm-ulm.de

Extended author information available on the last page of the article 


\section{Introduction}

Some of the most obvious changes during apple maturation concerns the pigmentation of the apple skin. Depending on the cultivar, the background coloration changes from green to yellow and the red blush color increases. The overall color impression is the combined result of several pigment types, which absorb the incident sunlight in one or more specific spectral bands. As in all higher plants, the chlorophylls in particular play an important role in photosynthesis by converting sunlight into chemical energy. Chlorophyll $a$ and chlorophyll $b$ have two distinct absorption bands, the more pronounced in the blue wavelength region (Soret band) and the second in the red wavelength region ( $\mathrm{Q}$ band), while the green components of the incident light are almost completely reflected [1]. The light reactions of photosynthesis take place in special cell organelles, the chloroplasts, whose thylakoid membrane contains various light-collecting protein complexes. Local stratification of the membrane results in the formation of disc-shaped structures called granules (grana) with a typical diameter of around $500 \mathrm{~nm}$ [2]. In addition to chlorophylls, various carotenoids, including carotenes (e.g., $\beta$-carotene) and xanthophylls (e.g., lutein), are involved in the light reaction in the thylakoid membrane [3]. The yellow coloration of apple skin, however, is determined by carotenoids independent of the photosynthetic system, e.g., xanthophylls such as lutein, neoxanthin and violaxanthin, which are esterified with fatty acids [4]. The third important pigment class is phenolic compounds, e.g., flavonoids and anthocyanins, which accumulate in cell vacuoles especially at the end of maturation [5]. They have a number of different functions, including photo-protection against harmful radiation in the ultraviolet (UV) and visible (Vis) spectral range or scavenging free radicals [6, 7]. Cyanidin 3-O-galactoside ('idaein') is the predominant anthocyanin found in apples, it absorbs light in the green spectral range and causes the intense red coloration of many apple cultivars [5]. In vacuoles, anthocyanins, together with other copigments, form complexes [8], now being referred to as AVIs, that are separated from the surrounding cell fluid but presumably have no membrane or distinct internal structure. All of these plant pigment types exhibit complex light interactions depending on their occurrence in particular cell organelles, although the specific effects on macroscopic absorption and scattering properties are still largely unknown.

In cell suspensions of green algae with high chlorophyll content, an early observed effect was selective scattering $[9,10]$ with a substantial increase in the effective scattering coefficient $\mu_{s}^{\prime}$ for the prominent absorption bands as associated with chlorophyll. Using Mie's theory and assuming simultaneously absorbing and scattering particles, indications of a correlation between the spectral course of absorption and scattering parameters with the structural properties of chloroplasts were found $[11,12]$. In this context, determining the complex refractive index for the pigment-containing organelles plays an important role. The complex refractive index is generally composed of a wavelength dependent real part (ratio between the speed of light in vacuum to the speed of light in a medium) and an imaginary part, which is directly linked to the absorption properties. Both parts are physically dependent on each other, so that in case of strong absorption, i.e., an increase of the imaginary part, the real part of the refractive index also changes in a certain wavelength range [13, 14]. For the chlorophyll-containing grana stacks in chloroplasts, recent studies have reconstructed the complex refractive index by considering their molecular structure and their influence on light propagation [15]. In particular, the influence of plant pigments on the bulk optical properties of apple has so far mainly been the result of experimental investigations, based on integrating sphere measurements. For example, Saeys et al. [16] and Rowe et al. [17] reported an increase in $\mu_{s}^{\prime}$ at spectral bands corresponding to high pigment and water absorption and attributed this to the increase in the imaginary part of the refractive index [18]. However, Zamora-Rojas et al. [19] proposed in a similar study examining animal tissue that the effect was more likely the result of a faulty separation between absorption and scattering and that increasing the imaginary part should not result in pronounced peaks in $\mu_{s}^{\prime}$. Thus, the influence of pigment-containing structures on the optical properties is still actively under discussion.

At the microscopic level, the interaction of light with grana and AVIs can be described approximately by scattering of spherical and absorbing particles within a non-absorbing surrounding medium. For this case, Mie's theory provides an analytical solution to Maxwell's equations, which gives exact results for arbitrary particle sizes and refractive index differences [20]. Model simulations recently described by Lohner et al. [21], developed to study the influence of air pores and starch granules on scattering in apple core and cortex tissues, could be adapted for pigment structures.

The aim of this work was to investigate the influence of pigment-containing organelles such as grana and AVIs on the optical properties of apple skin. Both an experimental and a theoretical approach were chosen each with the objective to determine $\mu_{a}$ and $\mu_{s}^{\prime}$ in the Vis spectral range. The application of Mie's theory for grana and AVIs required the reconstruction of their complex refractive indices, which was considered as an essential part of the modeling process. Additional measurements with an integrating sphere were intended to verify from an experimental point of view the extent to which differences in the optical properties of 
apple skin with either high chlorophyll or high anthocyanin content can be determined.

\section{Theoretical background}

\subsection{Mie's theory}

Mie's theory (also called Lorenz-Mie theory) is an analytical solution to Maxwell's equations and describes electromagnetic scattering by a homogeneous and isotropic sphere in a non-absorbing medium. The calculation is based on the size parameter $x=\pi d / \lambda$, as the ratio between sphere diameter $d$ and light wavelength $\lambda$, and the ratio $m$ between the complex refractive index $n_{\text {sph }}$ of the sphere and the real refractive index $n_{\text {med }}$ of the surrounding non-absorbing medium. Mie's theory provides exact solutions for the scattering coefficient $\mu_{s}$, the phase function $P(\theta, \phi)$, which describes the scattered light intensity in spherical coordinates, the anisotropy factor $g$, the effective scattering coefficient $\mu_{s}^{\prime}=(1-g) \mu_{s}$ and the absorption coefficient $\mu_{a}$ [20]. Since biological particles are usually subject to a broad size distribution, a polydisperse Mie model must be developed, in which the optical properties are averaged over different particle sizes, taking into account their volume fraction [22]. A detailed overview for the implementation using the MatScat function library [23] was recently presented by Lohner et al. [21]. It must be noted that for air and water, the absorption in the Vis is negligible, which means that in these cases the real part of the complex refractive index is sufficient for calculating the scattering properties. For pigment-containing particles, however, significant absorption has to be expected in this spectral range, which requires consideration of the complex refractive index.

\subsection{Reconstruction of the complex refractive index}

The refractive index plays an important role for light propagation in scattering media. In general, it is defined as complex number

$n_{\mathrm{sph}}=\eta+i \kappa$,

with a real part $\eta$ and an imaginary part $\kappa$. While the real part describes the ratio between the speed of light in vacuum and the speed of light in a medium, $\kappa$, also called extinction coefficient, defines the attenuation. The two parts are not completely independent of each other, but rather connected by Hilbert transforms, which are mathematically described by the Kramers-Kronig relations [24]. These are of particular importance for the investigation of biological tissue, since $\eta$ and $\kappa$ are usually only partially known or even not at all. In many cases, the real part can be determined experimentally, e.g. based on dispersion and refraction properties of a medium. The imaginary part is related to the absorption coefficient $\mu_{a}$ and the molar extinction coefficient $\epsilon_{M}$ according to

$\kappa(\lambda)=\frac{\mu_{a}(\lambda) \lambda}{4 \pi}=\frac{\ln 10 \epsilon_{M}(\lambda) c_{M} \lambda}{4 \pi}$,

with wavelength $\lambda$ and the molar concentration $c_{M}$ [25]. Even though estimates can be made independently for the real and imaginary parts, they must be linked via the Kramers-Kronig relations to maintain self-consistency. Especially in the wavelength range where strong absorption prevails, they lead to a physically reasonable adaptation of both parts. In this work, the function library presented by Lucarini et al. [24] was used with a numerical implementation of the Kramers-Kronig relations that provides the complex refractive index based on an estimate for $\kappa$ over the full spectral range from UV to IR and a single estimate for $\eta$.

\subsection{Chloroplasts and grana}

Apple skin has a mean thickness of about $100-150 \mu \mathrm{m}$ and can be divided into three different layers: the epidermis with cuticle on top and the hypodermis underneath [28]. With diameters in the range of $20-50 \mu \mathrm{m}$, the cell size of the epidermis and hypodermis is significantly smaller than that of the cortex tissue [29]. Their relatively dense cell structure is interrupted by many small air pores, which are important for gas exchange [30]. Photosynthesis takes place in certain cell organelles, the chloroplasts, which are found in large concentrations in apple skin [31,32]. Chloroplasts essentially contain an aqueous phase, the stroma, interspersed with thylakoid membranes. These form local layered structures called grana, which are connected by individual membrane layers of stromal thylakoids. Figure 1A shows schematically the structure of grana and their composition, which in reality can vary largely in shape and size. In higher plants, mostly cylindrical grana stacks of 10-20 membrane layers and dimensions of about $500 \mathrm{~nm} \times 300 \mathrm{~nm}$ are reported $[2,33]$. An enlarged view of two superimposed thylakoid layers and their substructure with dimensions reported by Daum et al. [26] is shown in Fig. 1B. The membranes enclose a further cavity of aqueous phase, the so-called lumen. The membrane itself contains 20\% lipids and $80 \%$ photoactive proteins, notably photosystem II (PSII) and light-harvesting complex II (LHCII) [34]. Together, these proteins form the supercomplex PSII/LHCII, which is essential for photosynthesis. Both the size and molecular composition of this protein complex have been extensively studied [35, 36]. According to Wei et al. [27] for spinach leaves, it has a size of $26 \mathrm{~nm} \times 11 \mathrm{~nm} \times 14 \mathrm{~nm}$ and contains 80 chlorophyll $a, 25$ chlorophyll $b$ and 28 carotenoid 


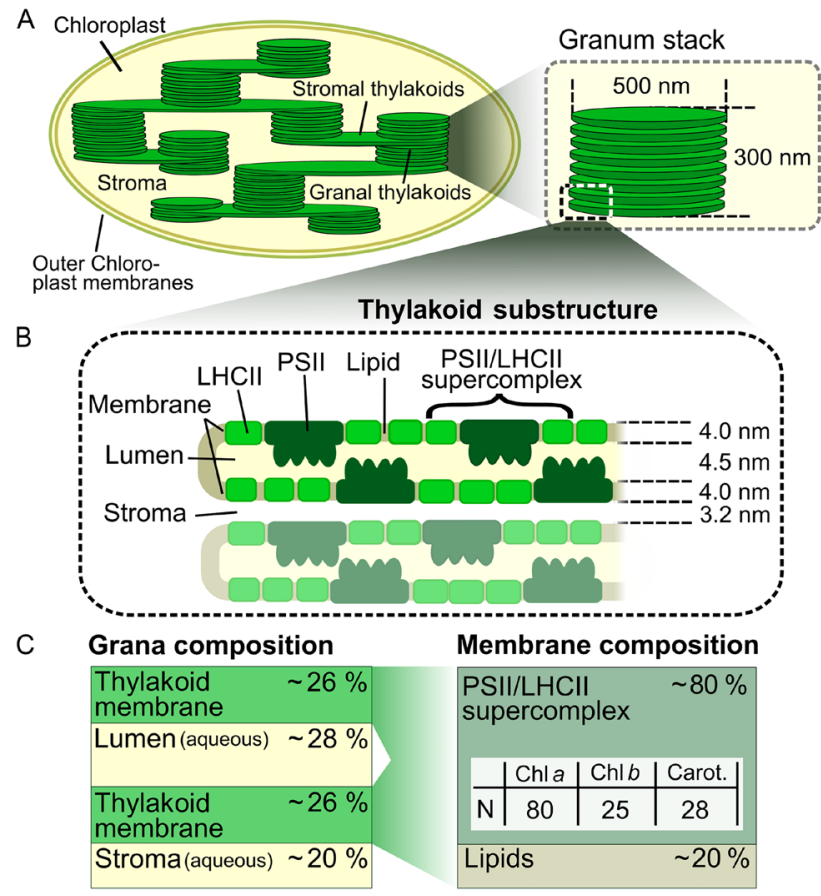

Fig. 1 A Schematic illustration of a chloroplast consisting of aqueous phase (stroma) and thylakoid membranes forming cylindrical stacked structures (grana) with typical sizes of $500 \mathrm{~nm} \times 300 \mathrm{~nm}$. B The thylakoid membrane encloses the lumen, also aqueous phase, and contains $20 \%$ lipids and $80 \%$ photoactive proteins. In particular, photosystem II (PSII) and the light-harvesting complex II (LHCII) are significantly involved in photosynthesis. The dimensions are taken from Daum et al. [26]. In $\mathbf{C}$, the volume fractions of the individual components are shown in an overview, for the PSII/LHCII complex indicating the number of molecules $N$ of chlorophyll $a$, chlorophyll $b$, and carotenoids [27]

molecules, resulting in molar concentrations of $0.063 \mathrm{~mol} / \mathrm{l}$, $0.020 \mathrm{~mol} / \mathrm{l}$ and $0.022 \mathrm{~mol} / \mathrm{l}$, respectively. As shown in an overview in Fig. 1C, approximately $48 \%$ of the grana volume is accounted for by the aqueous phase and $52 \%$ by the thylakoid membrane, which in turn consists of $80 \%$ pigmentcontaining protein complex and $20 \%$ lipids.

\subsection{Anthocyanic vacuolar inclusions (AVIs)}

In contrast to the rather well known structure of chloroplasts and grana, relatively little is known about anthocyanincontaining structures in apple skin. These can be found in the aqueous vacuoles along with various sugars, organic acids, amino acids and other phenolic constituents [37]. Flavonols such as quercetin-3-O-galactoside ('hyperoside') or quercetin-3-O-rutinoside ('rutin') with absorption in the near UV range are among the most abundant phenolics ahead of anthocyanins, in particular idaein, with an absorption maximum in the green spectral range [38]. Anthocyanins have the ability to form molecular non-covalent bonds depending on various external factors, which allows the

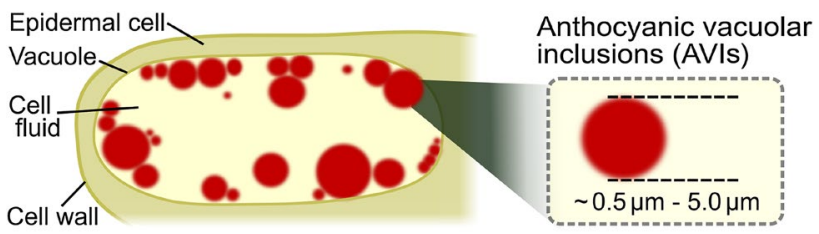

Fig. 2 Schematic illustration of an epidermal cell vacuole with AVIs having an average size between 0.5 and $5.0 \mu \mathrm{m}$

formation of anthocyanin complexes. Of particular importance are $\pi$-stacking interactions of aromatic rings underlying the mechanisms of self-association, i.e., binding between anthocyanin molecules, and co-pigmentation, i.e., binding of anthocyanin molecules with other phenols [39]. Complex formation is often favored in an acidic $\mathrm{pH}$ environment, resulting in significant differences in molar extinction bands, both spectrally and in absolute terms, between free and bound pigments [39]. Depending on the pigment type and $\mathrm{pH}$, both bathochromic and hypsochromic shifts of the absorption bands are observed, which explains the importance of these processes for color variation [39]. In cell vacuoles of apple skin, similar to other plants tending to have low $\mathrm{pH}$ in the vacuoles, the formation of so-called AVIs is reported [40], which can probably be regarded as a local accumulation of these anthocyanin complexes. According to Bae et al. [8], their diameter ranges from about 0.5 to $5.0 \mu \mathrm{m}$ for apple skin, with generally high variability in shape and size, as schematically shown in Fig. 2. The observation that AVIs appear to have no outer membrane [8] also supports the interpretation of pigment complexes bound by intra- and intermolecular interactions. The dynamic formation of AVIs appears very complex, for example, Kallam et al. [41] found that the formation of AVIs is related to $\mathrm{pH}$, with the acidic environment in the $\mathrm{pH}$ range of 4-5 likely changing during maturation [42]. In addition, the influence of flavonols and interactions with other biopolymers such as pectin and glycosides, whose concentrations in the vacuole are subject to considerable changes during maturation, are also conceivable [39].

\section{Materials and methods}

\subsection{Integrating sphere setup}

To determine the optical properties of apple skin over a wide spectral range in the Vis, an integrating sphere setup recently described by Foschum et al. [43] and Bergmann et al. [44] was used. It consists of a 3D-printed and barium sulfate-coated sphere with an inner diameter of $150 \mathrm{~mm}$, combined with a halogen light source and two spectrometers: one for the VIS from 200 to $1100 \mathrm{~nm}$ (Maya2000Pro, 
Ocean Optics, USA) and one for the near-infrared (NIR) from 900 to $1700 \mathrm{~nm}$ (NIRQuest512-1.7, Ocean Optics, USA). The light source is a $100 \mathrm{~W}$ halogen lamp (Halostar Starlite, Osram, Germany). During the measurement process, the absolute reflectance and transmittance spectra of a sample with known thickness is recorded over a wide spectral range. The evaluation is based on Monte Carlo simulations taking into account sample geometry, refractive index, and anisotropy factor, and provides a lookup table to determine the corresponding $\mu_{s}^{\prime}$ and $\mu_{a}$. For sample preparation, a peeler was used to remove pieces of skin from both the sun and shade sides of the apples, and the remaining pulp was carefully removed completely with a scalpel. The thickness of the skin samples was determined individually with a micrometer screw and taken into account in the following evaluation, the arithmetic mean value was $0.22 \pm 0.07 \mathrm{~mm}$. Both skin pieces were measured in a cuvette between two N-BK7 glass slides (34-427, Edmund Optics, USA), taking care to completely cover the sample port, which has a diameter of $25 \mathrm{~mm}$. The addition of a few drops of water prevented the formation of air bubbles between the slides, which would lead to undesirable influences on the measurement process. The sample cuvette was measured from each side at two positions rotated by $180^{\circ}$, and the optical properties were then calculated as the arithmetic mean with the corresponding standard deviation from four individual measurements. The spectrally resolved refractive index and the geometry of the glass sides were taken into account accordingly in the evaluation.

\subsection{Fruit samples}

The examined apple samples are identical to those investigated by Lohner et al. [21, 45]. They were collected from the research orchard of the Kompetenzzentrum Obstbau Bodensee ( $\left.47^{\circ} 46^{\prime} 01.8^{\prime \prime} \mathrm{N} 9^{\circ} 33^{\prime} 30.3^{\prime \prime} \mathrm{E}\right)$ during the 2019 harvest season between July and November. The cultivars were Malus domestica 'Gala' (Simmons/Buckeye), 'Elstar' (P.C.P.), and 'Jonagold' (Novajo). The selection of cultivars and the measurement period covered the early to late harvest period with different ripening characteristics and thus variable pigment content. Six apples were picked weekly and measured with a laboratory setup on the sun and shade side of each apple with four repetitions.

\section{Results and discussion}

\subsection{Complex refractive indices of grana and AVIs}

The reconstruction of the complex refractive index of the grana and AVIs required an estimation of their absorption properties over a wide spectral range from UV to NIR. As an approach, the individual components and their volume fractions were estimated on the basis of literature data. The full absorption spectra were then calculated as a linear combination of corresponding reference absorption spectra, as shown in Fig. 3A, B.

According to Fig. 1C, about $48 \%$ of the grana consists of aqueous phase, for which the absorption of water was assumed [46]. Due to the excitation of electronic transitions, it absorbs light in the UV at wavelengths below $180 \mathrm{~nm}$, while in the Vis the absorption is negligible and increases in the NIR beyond a wavelength of $900 \mathrm{~nm}$. The remaining $52 \%$ of the grana volume is occupied by the thylakoid membrane, which contains the pigment-containing protein complexes and lipids. The absorption spectrum of the lipids was adapted from McHowat et al. [47], who reported an absorption peak at a wavelength of $203 \mathrm{~nm}$ with a molar extinction $\epsilon_{M}=2 \cdot 10^{4} \mathrm{M}^{-1} \mathrm{~cm}^{-1}$ for phospholipids commonly found in cell membranes. Another absorption band in the UV at $187 \mathrm{~nm}$ can be attributed to the excitation of peptide bonds, which are present in large numbers in the protein complex of the membrane [48]. It was adapted from Gienger et al. [25] with $\epsilon_{M}=4 \cdot 10^{4}$ $\mathrm{M}^{-1} \mathrm{~cm}^{-1}$. The pigments contained in the protein complex begin to absorb at about $200 \mathrm{~nm}$, with maxima known to be mainly located in the Vis. In addition to chlorophyll $a$ and chlorophyll $b, \beta$-carotene was chosen as an example for carotenoids because a molar extinction spectrum in diethyl ether was also available from Taniguchi and Lindsey $[49,50]$. In general, the absorption properties of the carotenoids are quite similar, at least from a qualitative point of view [51]. Chlorophyll $a$ and $b$ exhibit absorption bands at wavelengths of $429 \mathrm{~nm}$ and $453 \mathrm{~nm}$ with $\epsilon_{M}=1.1 \cdot 10^{5} \mathrm{M}^{-1} \mathrm{~cm}^{-1}$ and $\epsilon_{M}=2 \cdot 10^{4} \mathrm{M}^{-1} \mathrm{~cm}^{-1}$ (Soret bands), and at $661 \mathrm{~nm}$ and $644 \mathrm{~nm}$ with $\epsilon_{M}=8.6 \cdot 10^{5} \mathrm{M}^{-1}$ $\mathrm{cm}^{-1}$ and $\epsilon_{M}=5.6 \cdot 10^{5} \mathrm{M}^{-1} \mathrm{~cm}^{-1}$ (Q bands), respectively. The absorption spectrum of $\beta$-carotene solubilized in hexane shows three adjacent absorption bands at wavelengths of $429 \mathrm{~nm}, 451 \mathrm{~nm}, 477 \mathrm{~nm}$, where the maximum extinction is given as $\epsilon_{M}=1.4 \cdot 10^{5} \mathrm{M}^{-1} \mathrm{~cm}^{-1}$ [50]. It should be noted that the absorption bands of these pigments behave differently in the solvent than in the protein environment, both in absolute values and in their spectral position. As shown in Fig. 3A, the absorption spectrum of grana was calculated as the sum of the weighted reference spectra, where $\mu_{a}$ was calculated from the indicated molar extinction coefficients according to Eq. (2). The enlarged figure in the wavelength range from 400 to $750 \mathrm{~nm}$ allows the assignment between characteristic absorption bands to the individual pigments in more detail.

Figure 3B shows the analogous procedure for modeling the absorption spectrum of the AVIs. It was assumed that the anthocyanin complexes in the case of copigmentation can be approximately described by an aqueous solution 

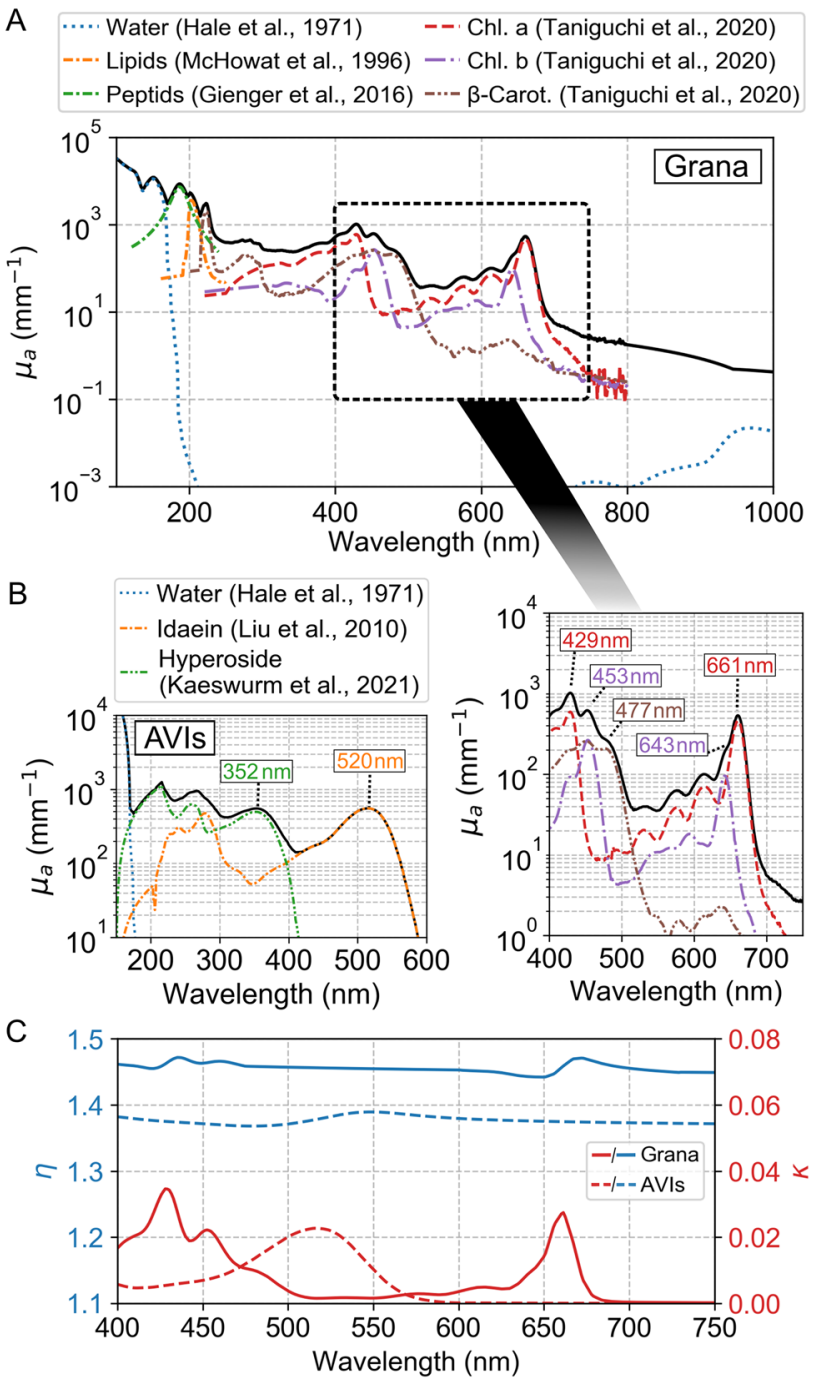

Fig. 3 A Reconstructed absorption coefficient $\mu_{a}$ of grana in the UV to Vis spectral region obtained by linear combination of weighted reference spectra considering aqueous phase, the thylakoid membrane composed of lipids, and the pigment-containing PSII/LHCII protein complex. The enlarged image section on the right below shows the Vis spectral region in detail with the Soret bands of chlorophyll $a$ and chlorophyll $b$ at $429 \mathrm{~nm}$ and $453 \mathrm{~nm}$, their Q bands at $643 \mathrm{~nm}$ and $661 \mathrm{~nm}$, overlaid by the absorption maxima of $\beta$-carotene with maximum at $453 \mathrm{~nm}$ and shoulder at $477 \mathrm{~nm}$. B Reconstructed $\mu_{a}$ of AVIs composed of water, hyperoside, and idaein with dominant absorption bands at $352 \mathrm{~nm}$ and $520 \mathrm{~nm}$, respectively. C Reconstructed complex refractive indices of grana and AVIs with real part $\eta$ (blue, upper curves) and imaginary part $\kappa$ (red, lower curves)

of $50 \mathrm{~mm}$ idaein and $100 \mathrm{~mm}$ hyperoside. The ratio of $2: 1$ for flavonols and anthocynanins was assumed regarding the results of Huber et al. [38] at the end of the maturation period. The absorption spectrum of idaein was taken from Liu et al. [52] and shows a broad absorption band with a maximum at a wavelength of $520 \mathrm{~nm}$. Following Fuleki and Francis [53], the absorption maximum was assumed to be $\epsilon_{M}=4.8 \cdot 10^{4} \mathrm{M}^{-1} \mathrm{~cm}^{-1}$. The absorption spectrum of hyperoside was taken from Kaeswurm et al. [54] and shows two smaller absorption bands with maximum wavelengths around $260 \mathrm{~nm}$ and $352 \mathrm{~nm}$, respectively. The spectrum was scaled to $\epsilon_{M}=2.2 \cdot 10^{4} \mathrm{M}^{-1} \mathrm{~cm}^{-1}$ at $352 \mathrm{~nm}$ [54]. It is noted that, contrary to this simplified assumption, in reality both the molar extinction and the spectral position of the absorption bands probably change during complex formation.

Based on the reconstructed absorption spectra, the imaginary parts $\kappa$ of the complex refractive indices were calculated using Eq. (2). The real parts $\eta$ were then computed using the function library provided by Lucarini et al. [24]. To compensate for the unavoidable errors caused by uncertainties in the estimation of the imaginary part, it proved useful to provide an estimate for the real part. For grana, a refractive index of 1.42 was chosen according to Margalit et al. [55]. Since AVIs do not have a distinct structure, a lower refractive index of 1.38 seems plausible. The results for self-consistent real and imaginary parts according to Kramers-Kronig relations are shown in Fig. 3C. Whereas $\kappa$ widely corresponds to the modeled absorption properties, the real part $\eta$ shows anomalous dispersion in the region of high absorption, i.e., the refractive index increases in these regions with increasing wavelength. This behavior leads simultaneously to a local increase of $\eta$ by about $3 \%$ with the spectral maximum shifted to longer wavelengths relative to the absorption band. In the case of grana, $\kappa$ shows a maximum at $429 \mathrm{~nm}$ with 0.035 and at $661 \mathrm{~nm}$ with 0.028 , while $\eta$ is in the range of 1.45 . In the case of AVIs, $\kappa$ reaches a maximum of 0.023 at $520 \mathrm{~nm}$, while $\eta$ lies in the range of 1.38 .

For comparison, the results of Capretti et al. [15] were considered, who similarly modeled the complex refractive index of grana. For the $\mathrm{Q}$ band of chlorophyll $a$ they reported also anomalous dispersion with $\kappa=0.014$ combined with a local change of $\eta$ by up to 5\%. Despite differences in the assumed composition and reference spectra used for modeling, the magnitude of the obtained complex refractive index could thus be confirmed. The correct application of the Kramers-Kronig relations, which are certainly subject to considerable uncertainty in the case of heterogeneous structures and only partly known absorption properties, has a major influence on the results. This is particular evident in a less pronounced anomalous dispersion in our results despite the comparatively higher extinction. Generally, it must be taken into account that the assumptions are based on results of different studies and therefore correspond only to a limited extent to the conditions for real chloroplasts in apple skin. The composition and content of different chlorophyll and carotenoids as well as the structure of the grana itself may differ. Since quantitative absorption spectra of the investigated pigments are only available for different types of solvents, the characteristic absorption bands are spectrally shifted compared to the natural protein environment. Nevertheless, the modeled absorption spectra provide a good 
overview of the influence of the individual components and allow a qualitative assignment of characteristic absorption bands. In the case of grana in particular, it becomes clear that, in contrast to the widely known $\mathrm{Q}$ bands of chlorophyll in the red spectral range, several broad bands overlap at wavelengths below $500 \mathrm{~nm}$, whose local maxima can be assigned to the Soret bands of chlorophyll $a$ and $b$ and $\beta$ -carotene with increasing wavelengths.

\subsection{Simulated optical properties of grana and AVIs using a Mie model}

Based on the reconstructed complex refractive indices, a polydisperse Mie model was used to simulate the optical properties of grana and AVIs. In both cases, a log-normal size distribution was assumed, with a mean diameter for grana of $0.5 \mu \mathrm{m}$ and for AVIs of $2.5 \mu \mathrm{m}$ with a standard deviation of $0.1 \mu \mathrm{m}$ and $0.5 \mu \mathrm{m}$, respectively. The surrounding medium in both cases was assumed to be water with the refractive index reported by Hale and Querry [56]. In the absence of literature data on the volume concentrations of grana and AVIs in apple skin, these were estimated to be $0.5 \%$ and $5 \%$, respectively, which was considered as a realistic chlorophyll and anthocyanin content. For example, this volume concentration would correspond to $1.1 \mathrm{mg}$ of idaein per $1 \mathrm{~g}$ fresh weight apple skin. In view of the subsequent comparison with real measurements, it was taken into account that the scattering properties of the apple skin are influenced not only by the grana and AVIs but also by other scattering particles, such as small air pores of the intercellular space. These typically lead to a steady decrease in $\mu_{s}^{\prime}$ with increasing wavelength, which can be described by a power law. For the simulation of these additional scattering particles, air pores with an average size of $3 \mu \mathrm{m}$ and a porosity of $5 \%$ were assumed, considering microscopic and micro-CT measurements [28, 57].

The results of the simulations for $\mu_{s}, g, \mu_{s}^{\prime}$ and $\mu_{a}$ are shown in Fig. 4 compared for grana, AVIs, and air pores. For grana and AVIs, $\mu_{s}$ lies in the range of $2-6 \mathrm{~mm}^{-1}$, whereas for air pores it is about $20 \mathrm{~mm}^{-1}$ due to the much higher refractive index difference. Larger differences can also be observed for the anisotropy factor $g$, which ranges from 0.9 to 0.8 for grana, from 0.95 to 0.90 for AVIs, and from 0.90 to 0.94 for air pores. In Fig. $4 \mathrm{C}, \mu_{s}^{\prime}$ is shown for air pores in combination with grana or AVIs to realistically reproduce scattering in red and green apple skin. For comparison, scattering of solely air pores is also shown and follows a power law $\propto \lambda^{-0.58}$. In spectral regions with high absorption, several corresponding peaks also occur in $\mu_{s}^{\prime}$. For grana two small peaks at $437 \mathrm{~nm}$ and $461 \mathrm{~nm}$ correspond to the Soret bands of chlorophyll $a$ and $b$, respectively, while the more pronounced peak at $671 \mathrm{~nm}$ corresponds to the Q band of chlorophyll $a$. All three are shifted between 8 and $10 \mathrm{~nm}$

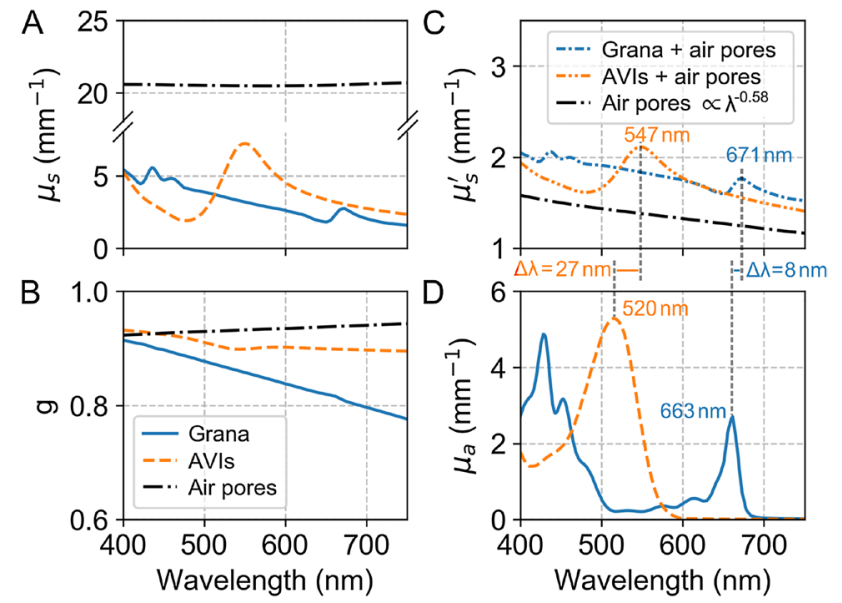

Fig. 4 Modeled scattering coefficients $\mu_{s}$ (A), anisotropy factors $g$ (B), effective scattering coefficients $\mu_{s}^{\prime}(\mathbf{C})$, and absorption coefficients $\mu_{a}$ (D) for grana, AVIs, and air pores with a mean size of $3 \mu \mathrm{m}$ in apple skin

to longer wavelengths relative to their associated absorption band. For AVIs, a broad increase of $\mu_{s}^{\prime}$ appears with maximum at $547 \mathrm{~nm}$ and a $27 \mathrm{~nm}$ red-shift of the central wavelength compared to idaein absorption.

When evaluating these results, it must be taken into account that the application of a Mie model requires different assumptions. The approximation of cell organelles as spherical particles seems evident in the case of the AVIs, whereas the grana have a more cylindrical shape. The expected deviations, however, are rather small at around 5\%, as shown by Grenfell et al. [58] using the example of cylindrical ice crystals with an unchanged volume-to-surface ratio. The assumed size distributions and volume concentrations of AVIs and grana, for which only sparse data are available, probably have a larger influence. For example, studies of Phan et al. [59, 60] showed that besides to small granules in apple skin, chloroplasts with few or even single, but larger granules are present in the pulp. Other microscopic studies of apple skin indicated an overall large variability in shape, size, volume concentration, and molecular composition of all types of organelles [8, 32]. The Mie model further assumes a homogeneous refractive index for the particles in the sense of an effective medium approximation, whereby changes in the refractive index due to the microstructure are not taken into account. Although this approximation is often used for biological tissue, its range of validity is difficult to estimate, especially for only roughly studied structures such as AVIs.

\subsection{Integrating sphere measurements to determine the optical properties of apple skin}

To experimentally investigate the influence of strongly absorbing pigments on the optical properties of apple skin, 
three different apple cultivars were measured with an integrating sphere during maturation. Over a period of ten weeks, a sample set of six apples with four measurement repetitions was examined weekly using samples from the sun side. The mean $\mu_{s}^{\prime}$ and $\mu_{a}$ and their standard deviation were determined by averaging both the results of the repetitions and all apples of the same cultivar on a weekly basis. The results are shown in Fig. 5 for the cultivars 'Elstar', with high chlorophyll content at the beginning of maturation, and
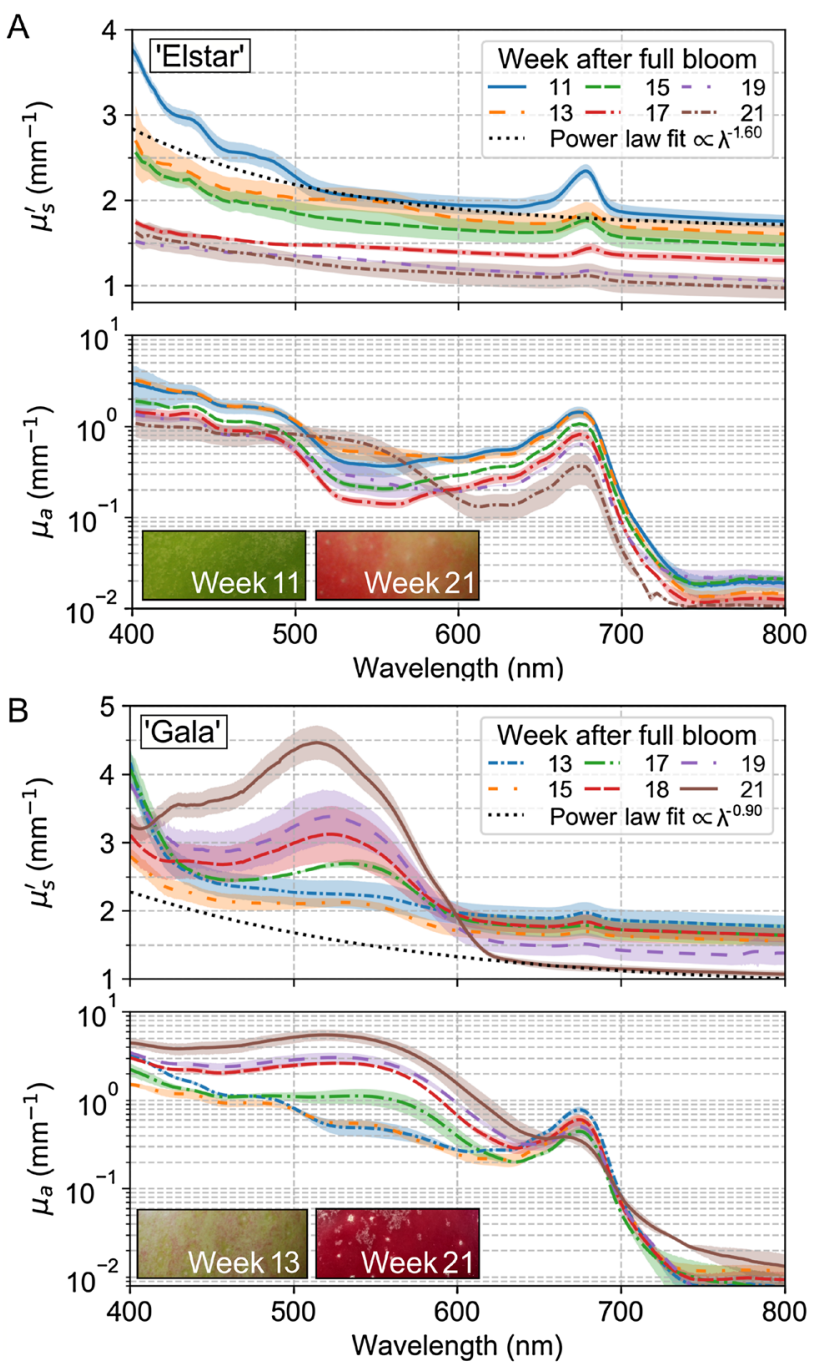

Fig. 5 Spectrally resolved effective scattering coefficient $\mu_{s}^{\prime}$ and absorption coefficient $\mu_{a}$ of apple skin measured for the cultivars 'Elstar' (A) and 'Gala' (B) during maturation. The shaded areas indicate the standard deviation based on a sample set of six apples per week with four measurement repetitions each. To determine the scattering not directly related to pigments, e.g., caused by air pores, a power law was fitted in spectral regions with negligible pigment absorption in $\mathbf{A}$ at week 11 and in $\mathbf{B}$ at week 21 after full bloom. The insets show color images of apple skin taken with an SLR camera from the first and last week of the maturation period studied to illustrate the cultivar-dependent change in coloration
'Gala' with a high anthocyanin content at the end of maturation. The insets show images of the skin color from the first and last week of the studied period.

For 'Elstar' in Fig. 5A, $\mu_{s}^{\prime}$ largely follows a power law of the form $\mu_{s}^{\prime}(\lambda) \propto \lambda^{-\alpha}$, i.e., $\mu_{s}^{\prime}$ decreases with increasing wavelength. In the first week, $\alpha=1.60$ was determined based on a regression. In the following weeks, $\mu_{s}^{\prime}$ shows a continuous decrease, e.g., at a wavelength of $600 \mathrm{~nm}$ from about $2.0 \mathrm{~mm}^{-1}$ in week 11 to $1.0 \mathrm{~mm}^{-1}$ in week 21 . Especially in week 11, $\mu_{s}^{\prime}$ shows several peaks in the wavelength range between 400 and $500 \mathrm{~nm}$ as well as at $678 \mathrm{~nm}$. These peaks appear to correspond to the absorption bands in $\mu_{a}$, with a peak at $678 \mathrm{~nm}$ known to be associated with chlorophyll $a$. It decreases from $1.38 \mathrm{~mm}^{-1}$ in week 11 to $0.36 \mathrm{~mm}^{-1}$ in week 21 . In the spectral range below $500 \mathrm{~nm}$, several smaller peaks stand out, with relatively high absorption between 1.0 and $3.0 \mathrm{~mm}^{-1}$. At the end of maturation in week $21, \mu_{a}$ increases in the range of about $520 \mathrm{~nm}$, which can be associated with anthocyanins and the corresponding slight red coloration.

Figure 5B shows the optical properties for the skin of 'Gala' apples, which exhibit a much more intense red coloration compared to 'Elstar'. Also in this case $\mu_{s}^{\prime}$ follows a power law in all weeks, with $\alpha=0.90$ determined in week 21. In addition, $\mu_{s}^{\prime}$ decreases from $2.1 \mathrm{~mm}^{-1}$ at week 13 to $1.1 \mathrm{~mm}^{-1}$ at week 21 at $650 \mathrm{~nm}$. Beginning at week 15, a broad increase occurs at a wavelength of $530 \mathrm{~nm}$, which increases steadily until the end of maturation. The difference between $\mu_{s}^{\prime}$ at $520 \mathrm{~nm}$ with $4.5 \mathrm{~mm}^{-1}$ and at $700 \mathrm{~nm}$ with $1.1 \mathrm{~mm}^{-1}$ at week 21 reaches about a factor of four. At the same time, $\mu_{a}$ also increases from 0.53 to $5.46 \mathrm{~mm}^{-1}$, which is due to the absorption of anthocyanins and causes dark red coloration. At $678 \mathrm{~nm}$, a small peak in $\mu_{s}^{\prime}$ is visible only in the first weeks of maturation with a corresponding decrease of $\mu_{a}$ from 0.76 to $0.33 \mathrm{~mm}^{-1}$.

To investigate the peaks in $\mu_{s}^{\prime}$ associated with either high chlorophyll or anthocyanin content, the optical properties for 'Elstar' from week 11 and 'Gala' from week 21 are shown in Fig. 6 in more detail. In both cases, the obtained power laws shown in Fig. 5 were subtracted from $\mu_{s}^{\prime}$. Without this background scattering, which is presumably caused by small air pores, the remaining fraction of $\mu_{s}^{\prime}$ can be attributed mainly to the influence of pigment-containing organelles. For apple skin with high chlorophyll content in Fig. 6A, four peaks can be identified at $435 \mathrm{~nm}, 469 \mathrm{~nm}, 488 \mathrm{~nm}$, and $678 \mathrm{~nm}$, whose central wavelengths were determined by regression of a Gaussian curve each. The two neighboring peaks at $469 \mathrm{~nm}$ and $488 \mathrm{~nm}$ can be distinguished only vaguely by a small dip. The height of the peaks relative to background is between 0.25 and $0.5 \mathrm{~mm}^{-1}$. The corresponding absorption spectrum shows increases of $\mu_{a}$ at corresponding spectral positions, but slightly shifted by about $4 \mathrm{~nm}$ to shorter wavelengths. It must be taken into account that apart from 

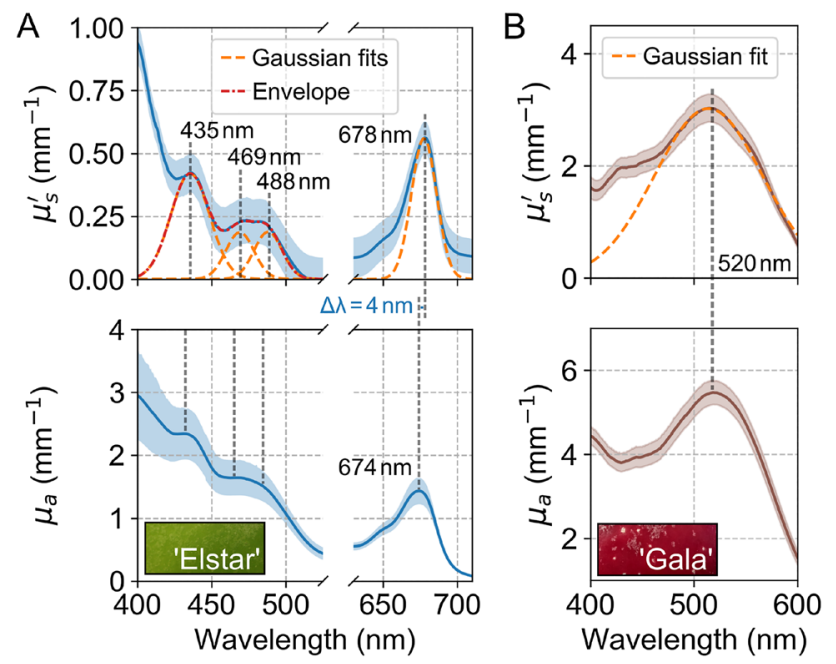

Fig. 6 Spectrally resolved optical properties of apple skin measured for 'Elstar' in week 11 (A) and 'Gala' in week 21 (B). In both $\mu_{s}^{\prime}$ spectra, the background was subtracted by means of the obtained power laws shown as black-dotted lines in Fig. 5. The indicated central wavelengths of the peaks as well as their spectral shift $\Delta \lambda$ were determined by regression of gaussian curves for $\mu_{s}^{\prime}$ and $\mu_{a}$

the carotenoids bound in the grana, other xanthophylls with almost the same absorption properties are present in the skin [4]. However, their concrete influence on the height and spectral position of the absorption bands is difficult to estimate and requires further investigation. For apple skin with high anthocyanin content in Fig. 6B, a single peak in $\mu_{s}^{\prime}$ with a height of up to $3 \mathrm{~mm}^{-1}$ relative to the subtracted background occurs. The corresponding absorption peak has a maximum of $6 \mathrm{~mm}^{-1}$ and no spectral shift was observed in this case.

In a comparable study using integrating sphere measurements, Van Beers et al. [61] observed a strong increase in $\mu_{s}^{\prime}$ at $550 \mathrm{~nm}$ during maturation from 1 to $5 \mathrm{~mm}^{-1}$ for two red-skinned cultivars, while no change was observed in 'Granny Smith' apples which are known to form almost no anthocyanins in the skin. This observation clearly confirms our results and thus the significant impact of AVIs on $\mu_{s}^{\prime}$. Naqvi et al. [62] presented a method to calculate the scattering and absorption properties based on extinction spectra of chloroplasts or LHCII solutions taking advantage of the Kramers-Kronig relations. In both cases, the results show qualitatively very similar characteristics compared to our measurement results, in particular pronounced selective scattering effects and a spectral offset between the absorption and scattering peaks.

Further comparison between these experimental results and our Mie simulations allows a more differentiated evaluation of the influence of grana and AVIs on the optical properties of apple skin. Both approaches independently demonstrated the occurrence of selective scattering effects in the spectral range of high chlorophyll and anthocyanin absorption. In contrast to the simulation results, in the experiment the peaks in $\mu_{s}^{\prime}$ are more pronounced relative to their corresponding $\mu_{a}$ band, and both are additionally spectrally shifted. Renge and Mauring [63] reported that chlorophyll absorption in protein environments is red-shifted by up to $20 \mathrm{~nm}$ compared to reference spectra in a solvent due to exciton interaction between closely spaced chromophores, which agrees well with our results for grana. The shift of the peaks in $\mu_{s}^{\prime}$ to longer wavelengths relative to $\mu_{a}$ is smaller than predicted, but confirms the presence of anomalous dispersion. Considering the explicit reconstruction of the complex refractive index, for grana the change in $\mu_{s}^{\prime}$ thus can be interpreted as the combined result of high extinction and local increase in the real part of the refractive index. Despite the spectral shift, the number and relative position of the peaks in $\mu_{s}^{\prime}$ agrees quite well with the model. Besides to the known $\mathrm{Q}$ band of chlorophyll $a$ at $678 \mathrm{~nm}$, by direct comparison between experiment and simulation the maxima at $435 \mathrm{~nm}$ and $469 \mathrm{~nm}$ can be assigned to the Soret bands of chlorophyll $a$ and $b$ and the maximum at $488 \mathrm{~nm}$ to the red-most absorption band of carotenoids. This assignment is well confirmed by the results of Croce et al. [64], who reported for the LHCII supercomplex the Soret bands of chlorophyll $a$ and $b$ at $439 \mathrm{~nm}$ and $467 \mathrm{~nm}$ and the red-most absorption bands of three predominant carotenoids, namely lutein, neoxanthin, and violaxanthin, at $489 \mathrm{~nm}, 488 \mathrm{~nm}$, and $492 \mathrm{~nm}$, respectively. The comparable $\mu_{a}$ for the maxima at $469 \mathrm{~nm}$ and $488 \mathrm{~nm}$ suggests a larger influence of carotenoids than the model predicts, either due to a higher molar extinction or, more likely, due to a higher molar concentration compared to the chlorophylls than assumed.

In the case of the AVIs, the increase in $\mu_{s}^{\prime}$ is almost an order of magnitude higher than predicted by the model, while the expected spectral shift between $\mu_{s}^{\prime}$ and $\mu_{a}$ is completely absent. Since only sparse information is available on the structure and chemical composition of AVIs, the reason for this cannot be conclusively determined. However, the absence of the spectral shift indicates that there is probably no abnormal dispersion and, accordingly, the reconstruction of the complex refractive index is not close to reality.

\subsection{Anthocyanin absorption shifts blue}

For the two cultivars 'Gala' and 'Jonagold' with intense red blush color at the end of maturation the anthocyanin absorption was investigated in more detail. In both cases, skin samples were taken weekly from the sun side of six apples and measured using the integrating sphere setup as previously presented. As shown in Fig. 7, their $\mu_{a}$ spectra show a dominant absorption peak that increases from about 1 to $6 \mathrm{~mm}^{-1}$ in less than 10 weeks. The central wavelength of the peaks, determined by regression of a Gaussian curve, shifts from an initial 
A

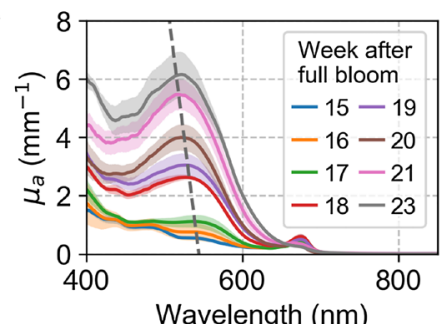

B

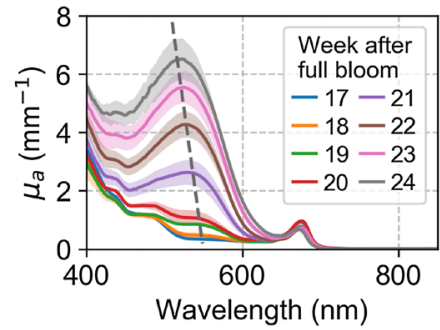

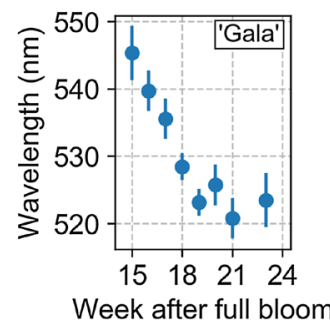

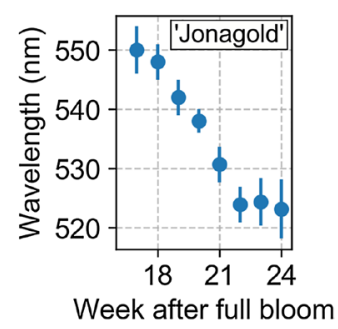

Fig. 7 Temporal evolution of the absorption coefficient $\mu_{a}$ in the Vis spectral range measured for the skin of A 'Gala' and $\mathbf{B}$ 'Jonagold' apples during maturation. The shaded area indicates the standard deviation of measurements on six different apples per week. On the right side, the central wavelength of the absorption band is plotted against the respective week after full bloom with error bars indicating the uncertainty of the underlying regression of a Gaussian curve

wavelength of about $550-520 \mathrm{~nm}$, over the same period. It is noticeable that both the absolute absorption and the shift are very similar when comparing the results of the two cultivars.

There are essentially two different explanations for the observed effect. On the one hand, the observed changes could be the result of an overlap with absorption bands of other pigments, which in principle could explain both the shift of the central wavelength and an apparent increase of $\mu_{a}$. Carotenoids in particular come into consideration, since for them an increase of $\mu_{a}$ in the spectral range up to about $500 \mathrm{~nm}$ at the end of maturation is documented [21, 65]. On the other hand, it must be taken into account that anthocyanins are generally chemically reactive due to their molecular structure, resulting in a large number of possible complex interactions. In particular, copigmentation plays an important role, meaning that anthocyanins form non-covalent bonds with other anthocyanins, other phenols such as flavonoids or even metal ions [66]. Under the influence of additional external factors, e.g., $\mathrm{pH}$ and temperature, both a bathochromic shift (red shift) and a hypsochromic shift (blue shift) in the central wavelength of the anthocyanin absorption as well as changes in the molar extinction coefficient can be observed [67]. According to Dangles et al. [39], the formation of AVIs can be understood as agglomeration of acylated anthocyanins. Thus, the observed blue shift would be a direct consequence of a chemical restructuring of the anthocyanins, which could possibly provide more detailed information about the structure of the AVIs or the $\mathrm{pH}$ in the cell vacuole.

\section{Conclusions}

Based on a combined theoretical and experimental study using apple skin as an example, the influence of grana and AVIs on the optical properties of biological tissue was described for the first time using quantitative quantities such as $\mu_{s}^{\prime}$ and $\mu_{a}$. The reconstruction of the absorption spectra showed that grana has characteristic absorption bands in the blue and red wavelength regions, which can be assigned to chlorophylls and carotenoids. In contrast, the AVIs showed a broad absorption band between 520 and $550 \mathrm{~nm}$. Consideration of the complex refractive indices based on the Kramers-Kronig relations together with a polydisperse Mie model allowed in an innovative way the prediction of multiple peaks in $\mu_{s}^{\prime}$ associated with the main absorption bands but shifted by $8-27 \mathrm{~nm}$ to longer wavelengths. This effect, known as selective scattering, was confirmed experimentally by measurements of apple skin samples with an integrating sphere, although differences in the height and spectral position of the peaks in $\mu_{a}$ and $\mu_{s}^{\prime}$ were found. By direct comparison, the Soret bands of chlorophyll $a$ and chlorophyll $b$ at $435 \mathrm{~nm}$ and $469 \mathrm{~nm}$, respectively, and at $488 \mathrm{~nm}$ the red-most edge of carotenoid absorption could be located. Especially for anthocyanin absorption, red-skinned apples showed a strong blue shift from 550 to $520 \mathrm{~nm}$ associated with an increase in absorption. While a spectral shift of about $4 \mathrm{~nm}$ between the peaks in $\mu_{a}$ and $\mu_{s}^{\prime}$ was found for grana in agreement with the Mie model, this was not the case for AVIs, raising further questions about their chemical structure and composition.

Overall, it was shown that simultaneously determining $\mu_{s}^{\prime}$ and $\mu_{a}$ provides complementary information about the chemical composition as well as structural parameters such as the size distribution of pigment-containing cell organelles or their refractive index difference relative to their surroundings. Due to the limited literature data specifically for apple skin, numerous assumptions had to be made in the modeling, which means that the comparison between experiment and theory in the context of this work is largely on a qualitative level. Nevertheless, it is conceivable to use the presented model on the basis of measured data also for quantitative evaluation, if individual aspects of the chemical composition or structure can be clarified in the future. For example, a regression model based on spectrally resolved $\mu_{s}^{\prime}$ and $\mu_{a}$ could be used to determine the size distribution of cell organelles for a given complex refractive index or vice versa. Facing the strong influence of AVIs on the optical properties, this approach could be useful to better understand the complex formation of anthocyanins, which are increasingly in focus due to their multiple physiological properties. In addition, the results are of interest in the growing field of applying optical properties for quality assurance of 
agricultural products, since high pigment concentrations in the skin and flesh of tomato, kiwi, or mango, for example, suggest comparable effects. In the long term, the development of microscopic light propagation models, e.g., based on numerical solutions to Maxwell's equations, would be desirable to obtain even more accurate and realistic results, especially with respect to heterogeneous cell organelles such as grana.

Acknowledgements This research was funded in part by 'Zentrales Innovationsprogramm Mittelstand' (ZIM) of the 'Bundesministerium für Wirtschaft und Energie' (BMWi).

\section{Declarations}

Conflict of interest On behalf of all authors, the corresponding author states that there is no conflict of interest.

\section{References}

1. Milne, B. F., Toker, Y., Rubio, A., \& Nielsen, S. B. (2015). Unraveling the intrinsic color of chlorophyll. Angewandte Chemie, 127(7), 2198-2201. https://doi.org/10.1002/ange.201410899

2. Mullineaux, C. W. (2005). Function and evolution of grana. Trends in Plant Science, 10(11), 521-525. https://doi.org/10.1016/j.tplan ts.2005.09.001

3. Hashimoto, H., Sugai, Y., Uragami, C., Gardiner, A. T., \& Cogdell, R. J. (2015). Natural and artificial light-harvesting systems utilizing the functions of carotenoids. Journal of Photochemistry and Photobiology C: Photochemistry Reviews, 25, 46-70. https://doi.org/10.1016/j.jphotochemrev.2015.07.004

4. Knee, M. (1988). Carotenol esters in developing apple fruits. Phytochemistry, 27(4), 1005-1009. https://doi.org/10.1016/ 0031-9422(88)80261-9

5. Lancaster, J. E., Grant, J. E., Lister, C. E., \& Taylor, M. C. (1994). Skin color in apples - influence of copigmentation and plastid pigments on shade and darkness of red color in five genotypes. Journal of the American Society for Horticultural Science, 119(1), 63-69. https://doi.org/10.21273/JASHS.119.1. 63

6. Merzlyak, M. N., \& Chivkunova, O. B. (2000). Light-stressinduced pigment changes and evidence for anthocyanin photoprotection in apples. Journal of Photochemistry and Photobiology B: Biology, 55(2-3), 155-163. https://doi.org/10.1016/S10111344(00)00042-7

7. Solovchenko, A., \& Schmitz-Eiberger, M. (2003). Significance of skin flavonoids for UV-B-protection in apple fruits. Journal of Experimental Botany, 54(389), 1977-1984. https://doi.org/10. 1093/jxb/erg 199

8. Bae, R.-N., Kim, K.-W., Kim, T.-C., \& Lee, S.-K. (2006). Anatomical observations of anthocyanin rich cells in apple skins. HortScience, 41(3), 733-736. https://doi.org/10.21273/HORTS CI.41.3.733

9. Latimer, P., \& Rabinowitch, E. (1959). Selective scattering of light by pigments in vivo. Archives of Biochemistry and Biophysics, 84(2), 428-441. https://doi.org/10.1016/0003-9861(59)90605-8

10. Merzlyak, M. N., et al. (2008). Light absorption and scattering by cell suspensions of some cyanobacteria and microalgae. Russian Journal of Plant Physiology, 55(3), 420-425. https://doi.org/10. 1134/S1021443708030199
11. Bialek, G. E., Horvath, G., Garab, G. I., Mustardy, L. A., \& Faludi-Daniel, A. (1977). Selective scattering spectra as an approach to internal structure of granal and agranal chloroplasts. Proceedings of the National Academy of Sciences, 74(4), 14551457. https://doi.org/10.1073/pnas.74.4.1455

12. Charney, E., \& Brackett, F. S. (1961). The spectral dependence of scattering from a spherical alga and its implications for the state of organization of the light-accepting pigments. Archives of Biochemistry and Biophysics, 92(1), 1-12. https://doi.org/10. 1016/0003-9861(61)90210-7

13. Chen, M., \& Weng, F. (2012). Kramers-Kronig analysis of leaf refractive index with the PROSPECT leaf optical property model. Journal of Geophysical Research: Atmospheres, 117(17), 1-9. https://doi.org/10.1029/2012JD017477

14. Sai, T., Saba, M., Dufresne, E. R., Steiner, U., \& Wilts, B. D. (2020). Designing refractive index fluids using the Kramers-Kronig relations. Faraday Discussions, 223, 136-144. https://doi.org/ $10.1039 / \mathrm{d} 0 \mathrm{fd} 00027 \mathrm{~b}$

15. Capretti, A., et al. (2019). Nanophotonics of higher-plant photosynthetic membranes. Light: Science and Applications, 8, 5. https://doi.org/10.1038/s41377-018-0116-8

16. Saeys, W., Velazco-Roa, M. A., Thennadil, S. N., Ramon, H., \& Nicolai, B. M. (2008). Optical properties of apple skin and flesh in the wavelength range from 350 to $2200 \mathrm{~nm}$. Applied Optics, 47(7), 908. https://doi.org/10.1364/AO.47.000908

17. Rowe, P. I., et al. (2014). Relationship between tissue firmness and optical properties of 'Royal Gala' apples from 400 to $1050 \mathrm{~nm}$. Postharvest Biology and Technology, 94, 89-96. https://doi.org/ 10.1016/j.postharvbio.2014.03.007

18. Bashkatov, A. N., Genina, E. A., Kochubey, V. I., \& Tuchin, V. V. (2005). Optical properties of human skin, subcutaneous and mucous tissues in the wavelength range from 400 to 2000 $\mathrm{nm}$. Journal of Physics D: Applied Physics, 38(15), 2543-2555. https://doi.org/10.1088/0022-3727/38/15/004

19. Zamora-Rojas, E., et al. (2013). Double integrating sphere measurements for estimating optical properties of pig subcutaneous adipose tissue. Innovative Food Science and Emerging Technologies, 19, 218-226. https://doi.org/10.1016/j.ifset.2013. 04.015

20. Bohren, C. F., \& Huffman, D. R. (1983). Absorption and scattering of light by small particles. New York: John Wiley.

21. Lohner, S. A., et al. (2021). Determining the optical properties of apple tissue and their dependence on physiological and morphological characteristics during fruit maturation. Part 2: Mie's theory. Postharvest Biology and Technology, 181, 111652. https:// doi.org/10.1016/j.postharvbio.2021.111652

22. Modest, M. F. (2003). Radiative heat transfer (2nd ed.). Boston: Academic Press. https://doi.org/10.1016/B978-0-12-503163-9. X5000-0

23. Schäfer, J. (2016). MatScat V1.4.0.0. MATLAB Central File Exchange. https://www.mathworks.com/matlabcentral/fileexchan ge/36831-matscat

24. Lucarini, V., Saarinen, J. J., Peiponen, K.-E., \& Vartiainen, E. M. (2005). Kramers-Kronig relations in optical materials research, vol. 110 of Springer Series in Optical Sciences. Berlin: SpringerVerlag. https://doi.org/10.1007/b138913

25. Gienger, J., Gross, H., Neukammer, J., \& Bär, M. (2016). Determining the refractive index of human hemoglobin solutions by Kramers-Kronig relations with an improved absorption model. Applied Optics, 55(31), 8951-8961. https://doi.org/10.1364/ao. 55.008951

26. Daum, B., Nicastro, D., Austin, J., Richard McIntosh, J., \& Kühlbrandt, W. (2010). Arrangement of photosystem II and ATP synthase in chloroplast membranes of spinach and pea. The Plant Cell, 22(4), 1299-1312. https://doi.org/10.1105/tpc.109.071431 
27. Wei, X., et al. (2016). Structure of spinach photosystem II-LHCII supercomplex at $3.2 \AA$ resolution. Nature, 534(7605), 69-74. https://doi.org/10.1038/nature 18020

28. Verboven, P., et al. (2013). Optical coherence tomography visualizes microstructure of apple peel. Postharvest Biology and Technology, 78, 123-132. https://doi.org/10.1016/j.postharvbio.2012. 12.020

29. Tukey, H. B., \& Young, J. O. (1942). Gross morphology and histology of developing fruit of the apple. Botanical Gazette, 104(1), 3-25. https://doi.org/10.1086/335103

30. Verboven, P., et al. (2008). Three-dimensional gas exchange pathways in pome fruit characterized by synchrotron X-ray computed tomography. Plant Physiology, 147(2), 518-527. https://doi.org/ 10.1104/pp.108.118935

31. Delgado-Pelayo, R., Gallardo-Guerrero, L., \& Hornero-Méndez, D. (2014). Chlorophyll and carotenoid pigments in the peel and flesh of commercial apple fruit varieties. Food Research International, 65, 272-281. https://doi.org/10.1016/j.foodres.2014.03.025

32. S. M, Schaeffer, et al. (2017). Comparative ultrastructure of fruit plastids in three genetically diverse genotypes of apple (Malus $\times$ domestica Borkh.) during development. Plant Cell Reports, 36(10), 1627-1640. https://doi.org/10.1007/s00299-017-2179-z

33. Mustárdy, L., \& Garab, G. (2003). Granum revisited. A threedimensional model - Where things fall into place. Trends in Plant Science, 8(3), 117-122. https://doi.org/10.1016/S1360-1385(03) 00015-3

34. Kirchhoff, H. (2008). Molecular crowding and order in photosynthetic membranes. Trends in Plant Science, 13(5), 201-207. https://doi.org/10.1016/j.tplants.2008.03.001

35. Nield, J., \& Barber, J. (2006). Refinement of the structural model for the photosystem II supercomplex of higher plants. Biochimica et Biophysica Acta - Bioenergetics, 1757(5-6), 353-361. https:// doi.org/10.1016/j.bbabio.2006.03.019

36. Dekker, J. P., \& Boekema, E. J. (2005). Supramolecular organization of thylakoid membrane proteins in green plants. Biochimica et Biophysica Acta - Bioenergetics, 1706(1-2), 12-39. https://doi. org/10.1016/j.bbabio.2004.09.009

37. Yamaki, S. (1984). Isolation of vacuoles from immature apple fruit flesh and compartmentation of sugars, organic acids, phenolic compounds and amino acids. Plant and Cell Physiology, 25(1), 151-166. https://doi.org/10.1093/oxfordjournals.pcp.a0766 88

38. Huber, G. M., \& Rupasinghe, H. P. (2009). Phenolic profiles and antioxidant properties of apple skin extracts. Journal of Food Science, 74(9), 693-700. https://doi.org/10.1111/j.1750-3841.2009. 01356. $\mathrm{x}$

39. Dangles, O., \& Fenger, J. A. (2018). The chemical reactivity of anthocyanins and its consequences in food science and nutrition. Molecules. https://doi.org/10.3390/molecules23081970

40. Zhang, H., Wang, L., Deroles, S., Bennett, R., \& Davies, K. (2006). New insight into the structures and formation of anthocyanic vacuolar inclusions in flower petals. BMC Plant Biology, 6, 1-14. https://doi.org/10.1186/1471-2229-6-29

41. Kallam, K., et al. (2017). Aromatic decoration determines the formation of anthocyanic vacuolar inclusions. Current Biology, 27(7), 945-957. https://doi.org/10.1016/j.cub.2017.02.027

42. Richmond, A. E., Dilley, D. R., \& Dewey, D. H. (1964). Cation, organic acid, and $\mathrm{pH}$ relationships in peel tissue of apple fruits affected with Jonathan spot. Plant Physiology, 39(6), 1056-1060. https://doi.org/10.1104/pp.39.6.1056

43. Foschum, F., Bergmann, F., \& Kienle, A. (2020). Precise determination of the optical properties of turbid media using an optimized integrating sphere and advanced Monte Carlo simulations. Part 1: theory. Applied Optics, 59(10), 3203. https://doi.org/10.1364/AO. 386011
44. Bergmann, F., Foschum, F., Zuber, R., \& Kienle, A. (2020). Precise determination of the optical properties of turbid media using an optimized integrating sphere and advanced Monte Carlo simulations. Part 2: experiments. Applied Optics, 59(10), 3216. https:// doi.org/10.1364/AO.385939

45. Lohner, S. A., et al. (2021). Determining the optical properties of apple tissue and their dependence on physiological and morphological characteristics during maturation. Part 1: spatial frequency domain imaging. Postharvest Biology and Technology, 181, 111647. https://doi.org/10.1016/j.postharvbio.2021.111647

46. Segelstein, D. J. (1981). The complex refractive index of water. Ph.d. thesis, University of Missouri - Kansas City.

47. McHowat, J., Jones, J. H., \& Creer, M. H. (1996). Quantitation of individual phospholipid molecular species by UV absorption measurements. Journal of Lipid Research, 37(11), 2450-2460. https://doi.org/10.1016/S0022-2275(20)37493-9

48. Woods, A. H., \& O'Bar, P. R. (1970). Absorption of proteins and peptides in the far ultraviolet. Science, 167(3915), 179-181. https://doi.org/10.1126/science.167.3915.179

49. Taniguchi, M., \& Lindsey, J. S. (2021). Absorption and fluorescence spectral database of chlorophylls and analogues. Photochemistry and Photobiology, 97(1), 136-165. https://doi.org/10. 1111/php.13319

50. Taniguchi, M., \& Lindsey, J. S. (2018). Database of absorption and fluorescence spectra of $>300$ common compounds for use in photochem CAD. Photochemistry and Photobiology, 94(2), 290-327. https://doi.org/10.1111/php.12860

51. Solovchenko, A. (2010). Screening pigments: general questions. In: Photoprotection plants. Springer Ser. Biophys. vol 14. Berlin: Springer. https://doi.org/10.1007/978-3-642-13887-4_2

52. Liu, P., Yang, B., \& Kallio, H. (2010). Characterization of phenolic compounds in Chinese hawthorn (Crataegus pinnatifida Bge. var. major) fruit by high performance liquid chromatography-electrospray ionization mass spectrometry. Food Chemistry, 121(4), 1188-1197. https://doi.org/10.1016/j.foodchem.2010.02. 002

53. Fuleki, T. ., \& Francis, F. .J. (1968). Quantitative methods for anthocyanins. 2. Determination of total anthocyanin and degradation index for cranberry juice. Journal of Food Science, 33(1), 78-83. https://doi.org/10.1111/j.1365-2621.1968.tb00888.x

54. Kaeswurm, J. A., Scharinger, A., Teipel, J., \& Buchweitz, M. (2021). Absorption coefficients of phenolic structures in different solvents routinely used for experiments. Molecules, 26(15), 1-15. https://doi.org/10.3390/molecules26154656

55. Margalit, O., Sarafis, V., \& Zalevsky, Z. (2010). The effect of grana and inter-grana components of chloroplasts on green light transmission: a preliminary study. Optik, 121(16), 1439-1442. https://doi.org/10.1016/j.ijleo.2009.02.007

56. Hale, G. M., \& Querry, M. R. (1973). Optical constants of water in the 200-nm to $200-\mu \mathrm{m}$ wavelength region. Applied Optics, 12 (3), 555. https://doi.org/10.1364/AO.12.000555

57. Janssen, S., et al. (2020). 3D pore structure analysis of intact 'Braeburn' apples using X-ray micro-CT. Postharvest Biology and Technology, 159, 111014. https://doi.org/10.1016/j.postharvbio. 2019.111014

58. Grenfell, T. C., \& Warren, S. G. (1999). Representation of a nonspherical ice particle by a collection of independent spheres for scattering and absorption of radiation. Journal of Geophysical Research: Atmospheres, 104(D24), 31697-31709. https://doi.org/ 10.1029/1999JD900496

59. Phan, C. T. (1973). Chloroplasts in the peel and internal apple tissue. Experientia, 29, 1555-1557.

60. Phan, C. T. (1984). All-granal chloroplasts of apple-fruit. Advances in Photosynthesis Research, III, 63-66. https://doi.org/ 10.1007/978-94-017-4973-2_14 
61. Van Beers, R., et al. (2017). Effect of maturation on the bulk optical properties of apple skin and cortex in the 500-1850 nm wavelength range. Journal of Food Engineering, 214, 79-89. https:// doi.org/10.1016/j.jfoodeng.2017.06.013

62. Naqvi, K. R., Merzlyak, M. N., \& Melø, T. B. (2004). Absorption and scattering of light by suspensions of cells and subcellular particles: an analysis in terms of Kramers-Kronig relations. Photochemical and Photobiological Sciences, 3(1), 132-137. https:// doi.org/10.1039/b304781d

63. Renge, I., \& Mauring, K. (2013). Spectral shift mechanisms of chlorophylls in liquids and proteins. Spectrochimica Acta Part A: Molecular and Biomolecular Spectroscopy, 102, 301-313. https:// doi.org/10.1016/j.saa.2012.10.034

64. Croce, R., Cinque, G., Holzwarth, A. R., \& Bassi, R. (2000). The Soret absorption properties of carotenoids and chlorophylls in antenna complexes of higher plants. Photosynthesis Research, 64(2-3), 221-231. https://doi.org/10.1023/A:1006455230379
65. Ampomah-Dwamena, C., et al. (2012). Metabolic and gene expression analysis of apple (Malus $\times$ domestica) carotenogenesis. Journal of Experimental Botany, 63(12), 4497-4511. https://doi. org/10.1093/jxb/ers134

66. Pina, F., Melo, M. J., Laia, C. A., Parola, A. J., \& Lima, J. C. (2012). Chemistry and applications of flavylium compounds: a handful of colours. Chemical Society Reviews, 41(2), 869-908. https://doi.org/10.1039/c1cs15126f

67. Giusti, M. M., Rodríguez-Saona, L. E., \& Wrolstad, R. E. (1999). Molar absorptivity and color characteristics of acylated and nonacylated pelargonidin-based anthocyanins. Journal of Agricultural and Food Chemistry, 47(11), 4631-4637. https://doi.org/10.1021/ jf981271k

\section{Authors and Affiliations}

\section{Stefan A. Lohner ${ }^{1}\left(\mathbb{D} \cdot\right.$ Konni Biegert $^{2} \cdot$ Ansgar Hohmann $^{1} \cdot$ Roy McCormick $^{2} \cdot$ Alwin Kienle $^{1}$}

$1 \quad$ Institut für Lasertechnologien in der Medizin und Meßtechnik an der Universität Ulm, Helmholtzstr. 12, 89081 Ulm, Germany
2 Kompetenzzentrum Obstbau Bodensee, Schuhmacherhof 6, 88213 Ravensburg, Germany 